\title{
Paper
}

\section{Development of Low Iron Loss Soft Magnetic Powder Core}

\author{
Hijiri TSURUTA*, Tomoyuki UENO and Kouji YAMADA \\ Sumitomo Electric Industries, Ltd., 1-1-1 Koya-kita, Itami 664-0016, Japan.
}

Received November 30, 2015; Revised February 29, 2016; Accepted March 3, 2016

\begin{abstract}
Recently, there's been a growing trend toward a low carbon society from rise on the interest of the environment. In the automotive industry, environmentally-friendly vehicles, like hybrid electric vehicles and plug-in hybrid electric vehicles, are replacing petrol vehicles. Due to this trend, the demand of power supply devices, such as converters, increase more and more. And there devices are required good conversion efficiency and downsizing. Therefore, these devices require the soft magnetic materials which have excellent alternative current (AC) magnetic property.

The authors have developed the soft magnetic powder cores which are made by compacting soft magnetic powder coated with the insulation film and then heat-treating to remove the compacting strain. The cores have high performance of AC magnetic property, low iron loss and high flux density. In this study, the effect of particle size distribution on the permeability at a high magnetic field and iron loss of FeSiAl alloy soft magnetic powder core was reported from the view point of demagnetizing coefficient.
\end{abstract}

\section{KEY WORDS}

soft magnetic material, soft magnetic powder core, choke coil

\section{Introduction}

Recently, there's been a growing trend toward a low carbon society from rise on the interest of the environment. In the automotive industry, environmentally-friendly vehicles, like hybrid electric vehicles and plug-in hybrid electric vehicles, are replacing petrol vehicles. The components for electric drive, such as motors or transformers, are composed iron cores consist of soft magnetic material, copper windings, and so on. Some of the representative soft magnetic materials include ferrites and lamination steel sheets. For ferrites, iron loss is low at a wide frequency range, but magnetic flux density is low. For lamination steel, iron loss is bad at high frequency, but magnetic flux density is high. Therefore, soft magnetic powder core is getting a lot of attention as the martial which have both characteristics in recent years.

One of the promising uses of soft magnetic powder core is choke coil for $\mathrm{DC} / \mathrm{DC}$ converter. Since it is driven in the high frequency range about $100 \mathrm{kHz}$, ferrites have been used for iron core so far. In recent years, owing to a reduction in the withstand voltage associated with the downsizing of semiconductor elements, the demand for equipment with large current operating has increased. The material is required high permeability at high magnetic field, for large current operating. However, ferrites have the problem that the permeability

* Corresponding author, E-mail: tsuruta-hijiri@sei.co.jp is low at high magnetic field, as its magnetic flux density is low. So, iron cores are required to become large to use in this state, therefore choke coil also become large.

In regard to above problem, we set out to development of iron core which is small and can be used at large current, by applying the soft magnetic powder cores which have high permeability at high magnetic field and low iron loss. And by development of FeSiAl soft magnetic powder core, we improved iron loss as same level as ferrite and increased permeability at high magnetic field than ferrite ${ }^{1)}$.

This paper reports the influence of particle size distribution on iron loss and permeability at high magnetic field, intended to improve the permeability of developed material at high magnetic field furthermore.

\section{Experimental Procedure}

Fe-8.5 wt $\%$ Si-6.8 wt $\%$ Al gas-atomized powder with particle sizes under $106 \mu \mathrm{m}$ was used, and three types of powders with $22 \mathrm{wt} \%, 30 \mathrm{wt} \%$ and $39 \mathrm{wt} \%$ ratios of particle sizes under $30 \mu \mathrm{m}$ were prepared. The powder particles were coated with silicates as an insulating layer. After that, $0.5 \mathrm{wt} \%$ PVA was added to the powder as a binder, and which was compacted to ring shape of $\varnothing 34 \times \varnothing 20 \times 5 \mathrm{~mm}$ with a density of $5.6 \mathrm{~g} / \mathrm{cm}^{3}$. Following this, the compacts were heat-treated at $973 \mathrm{~K}$ in air atmosphere. 
Iron loss was measured at room temperature at $10-100 \mathrm{kHz}$ and $0.1 \mathrm{~T}$ by using AC-BH analyzer (SY 8258; Iwatsu electric co., ltd). The magnetic field dependency of permeability was calculated from the DC magnetization characteristics. The DC magnetization characteristics were measured to be up to $16 \mathrm{kA} / \mathrm{m}$ using DC-BH analyzer.

\section{Results and Discussion}

\subsection{Iron loss properties}

Fig. 1 shows the measurement results for iron loss at $100 \mathrm{kHz}$. The iron loss improved with increase the ratio of particles under $30 \mu \mathrm{m}$ from $22 \mathrm{wt} \%$ to $39 \mathrm{wt} \%$. In general, with a decrease in particle sizes, the hysteresis loss increases due to coercive force increasing, and the eddy current loss decreases due to electric resistance increasing. Considering these factors, it is supposed that: the decrease of the eddy current loss is largely affected with the ratio of particles under $30 \mu \mathrm{m}$.

\subsection{Magnetic field dependency of permeability}

Fig. 2 shows the DC magnetization characteristics. The magnetic field dependency of permeability (Fig. 3) was calculated using the DC magnetization characteristics, as shown in Equation (1).

$$
\mu=\frac{1}{\mu_{0}} \times \frac{d B}{d H}
$$

Where, $\mu$ is differential permeability, $\mu_{0}$ is vacuum permeability, B is magnetic flux density, and $\mathrm{H}$ is the magnetic field. The magnetic field dependency of the permeability is almost same with increase of the ratio of particles under $30 \mu \mathrm{m}$ from $22 \mathrm{wt} \%$ to $30 \mathrm{wt} \%$. On the other hand, the permeability increases by approximately $3 \%$ at $16 \mathrm{kA} / \mathrm{m}$ with increase of the ratio from $30 \mathrm{wt} \%$ to $39 \mathrm{wt} \%$.

In this study, we discuss the main factor which improves the permeability.

According to the Ollendorf's formula, expressing the permeability of soft magnetic powder cores, the permeability $\mu$ was calculated using Equation $(2)^{2)}$.

$$
\mu=\frac{\eta \mu_{0}\left(\mu_{t}-\mu_{0}\right)}{\left[N(1-\eta)\left(\mu_{t}-\mu_{0}\right)+\mu_{0}\right]}+\mu_{0}
$$

Where, $\eta$ is the packing density, $\mathrm{N}$ is the demagnetizing coefficient, $\mu_{\mathrm{t}}$ is intrinsic permeability of material, and $\mu_{0}$ is the vacuum permeability. However, this formula is insufficient because it cannot express the smooth magnetization curve as the measuring curve.

In order to solve above problem, Saito et al. reported that the center value of the demagnetizing coefficient and its distribution affect the shape of the DC magnetization characteristics ${ }^{3}$. The center value of the demagnetizing coefficient $\mathrm{N}$ can be calculated using Equation $(3)^{4)}$, and the standard deviation of the demagnetizing coefficient $\sigma_{\mathrm{N}}$ is shown by Equation (4) ${ }^{3)}$.

$$
N=\frac{\delta}{D} \times N p
$$

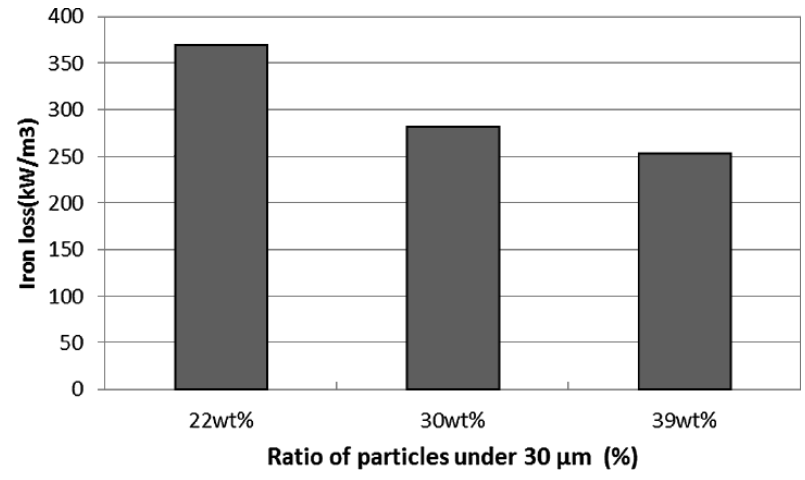

Fig. 1 Iron loss properties at $100 \mathrm{kHz}$.

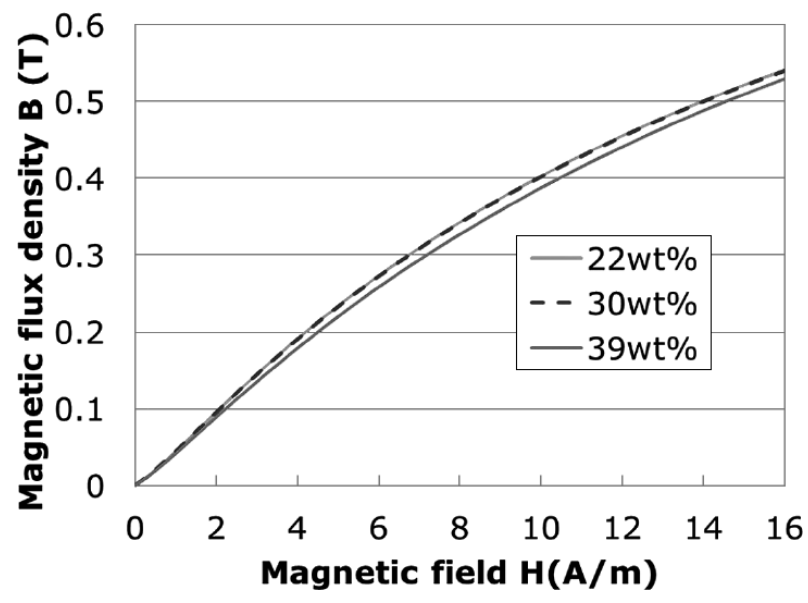

Fig. 2 DC magnetization characteristics.

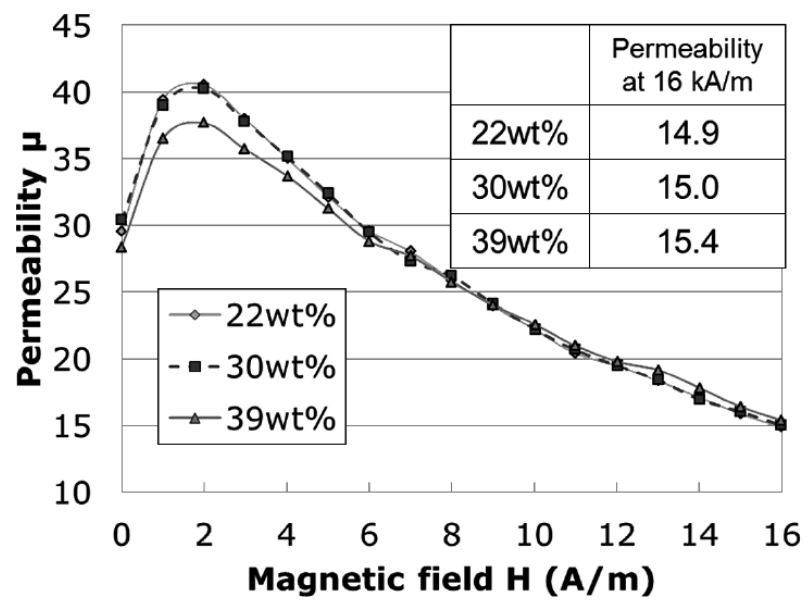

Fig. 3 The magnetic field dependency of permeability.

$$
\left(\log _{10} \sigma_{\mathrm{N}}\right)^{2}=\left(\log _{10} \sigma_{\mathrm{D}}\right)^{2}+\left(\log _{10} \sigma_{\delta}\right)^{2}+\left(\log _{10} \sigma_{\mathrm{Np}}\right)^{2}
$$

Where, $\delta$ is the gap distance, D is the particle diameter, and $\mathrm{Np}$ is demagnetizing coefficient of particle. $\mathrm{Np}$ is calculated using the aspect ratio m, as shown in Equation (5) ${ }^{5}$.

$$
N p=1 / 2\left[\left\{m^{2} /\left(m^{2}-1\right)\right\}^{\frac{3}{2}} \sin ^{-1}\left\{\left(m^{2}-1\right)^{\frac{1}{2}} / m\right\}-\left\{1-\left(m^{2}-1\right)\right\}\right]
$$


Table 1 The center values and the standard deviations for each factor.

\begin{tabular}{ccccc}
\hline \multicolumn{2}{c}{ Ratio of particles under $30 \mu \mathrm{m}(\mathrm{wt} \%)$} & 22 & 30 & 39 \\
\hline \multirow{2}{*}{ Demagnetizing coefficient of particle } & $\mathrm{Np}$ & 0.28 & 0.26 & 0.27 \\
& $\sigma_{\mathrm{Np}}$ & 0.04 & 0.05 & 0.05 \\
\hline \multirow{2}{*}{ Particle diameter $(\mu \mathrm{m})$} & $\mathrm{D}$ & 0.28 & 0.27 & 0.31 \\
& $\sigma_{\mathrm{D}}$ & 0.21 & 0.17 & 0.18 \\
\hline \multirow{2}{*}{ Gap distance $(\mu \mathrm{m})$} & $\delta$ & 54.0 & 50.6 & 45.8 \\
& $\sigma_{\delta}$ & 27.8 & 29.1 & 30.2 \\
\hline
\end{tabular}

We examine the main factor which improves the permeability by measuring these factors affecting the demagnetizing coefficient. Aspect ratio and $\delta$ were measured by image analysis using the cross sectional images, and D were measured by the laser diffraction method. Np was calculated using the measured aspect ratio and Equation (5). Table 1 shows the center values and the standard deviations for each factor.

Further, the relationship between the ratio of particles under $30 \mu \mathrm{m}$, and the center values or the standard deviations calculated from Table 1, Equation (3) and Equation (4) are shown in Fig. 4. The center value of the demagnetizing coefficient are almost same with increase the ratio of particles under $30 \mu \mathrm{m}$ from $22 \mathrm{wt} \%$ to $30 \mathrm{wt} \%$, however, the center value of the demagnetizing coefficient increases with increase the ratio from $30 \mathrm{wt} \%$ to $39 \mathrm{wt} \%$. This corresponds to the trend of permeability in relation to the ratio of particles under $30 \mu \mathrm{m}$. On the other hand, the standard deviations for the demagnetizing coefficient are almost same in relation to the ratio of particles under $30 \mu \mathrm{m}$. From the above results, it can be said that the center value of the demagnetizing coefficient exerts a dominant effect on the change of permeability in relation to the ratio of particles under $30 \mu \mathrm{m}$.

Furthermore, it will be identified which factors that exert a significant effect on the change of center value of the demagnetizing coefficient. The change in the center value of the demagnetizing coefficient can be expressed as the product of factors which are the gap distance $\delta$, the reciprocal of particle diameter $1 / \mathrm{D}$, and the particle demagnetizing coefficient $\mathrm{Np}$, as shown in Equation (3). So the main factor which strongly affects the change in center value of the demagnetizing coefficient can be examined with their
Table 2 The ratio of change of each factor in relation to the change of ratio of particles under $30 \mu \mathrm{m}$

\begin{tabular}{ccc}
\hline Sample & \multicolumn{2}{c}{ Ratio of change in each Factor } \\
\cline { 2 - 3 } & $22 \rightarrow 30 \mathrm{wt} \%$ & $30 \rightarrow 39 \mathrm{wt} \%$ \\
\hline Center value of demagnetizing coefficient $\mathrm{N}$ & $-0.3 \%$ & $27.6 \%$ \\
Demagnetizing coefficient of particle Np & $-5.0 \%$ & $2.7 \%$ \\
Particle diameter 1/D $\left(\mu \mathrm{m}^{-1}\right)$ & $6.7 \%$ & $10.5 \%$ \\
Gap distance $\delta(\mu \mathrm{m})$ & $-1.6 \%$ & $12.5 \%$ \\
\hline
\end{tabular}

change ratio. Table 2 shows the ratio of change of each factor in relation to the change of ratio of particles under $30 \mu \mathrm{m}$. No change in the center value of the demagnetizing coefficient with increase the ratio of particles under $30 \mu \mathrm{m}$ from $22 \mathrm{wt} \%$ to $30 \mathrm{wt} \%$ was observed, as the effect of the decrease in the $\mathrm{D}$ is compensated by the effects of the decrease in the particle demagnetizing coefficient and in $\delta$. The increase in the center value of the demagnetizing coefficient, or that is improvement of permeability, with increase the ratio of particles under $30 \mu \mathrm{m}$ from $30 \mathrm{wt} \%$ to $39 \mathrm{wt} \%$ was affected the change in the $\mathrm{D}$ and the $\delta$ strongly as compared to the particle demagnetizing coefficient.

Based on the relationship between the powder particle distribution and iron loss (Fig. 1), it is supposed that the core can get lower iron loss and high permeability at a high magnetic field by the increase in the ratio of particles under $30 \mu \mathrm{m}$.

\section{Summary}

In this study, in order to find out the possibility of improvement of permeability of the low-loss FeSiAl soft magnetic powder core, the effect of particle size on the permeability and iron loss of this material at a high magnetic field was examined from the view point of demagnetizing coefficient. The results are summarized as follows:

- It is confirmed the modification of the powder particle distribution improve the iron loss and the permeability at a high magnetic field.

- With regard to the change in permeability, the change in center value of the demagnetizing coefficient exhibits a dominant effect.

- The change in center value of the demagnetizing coefficient is largely affected by the change in particle diameter and the gap distance.
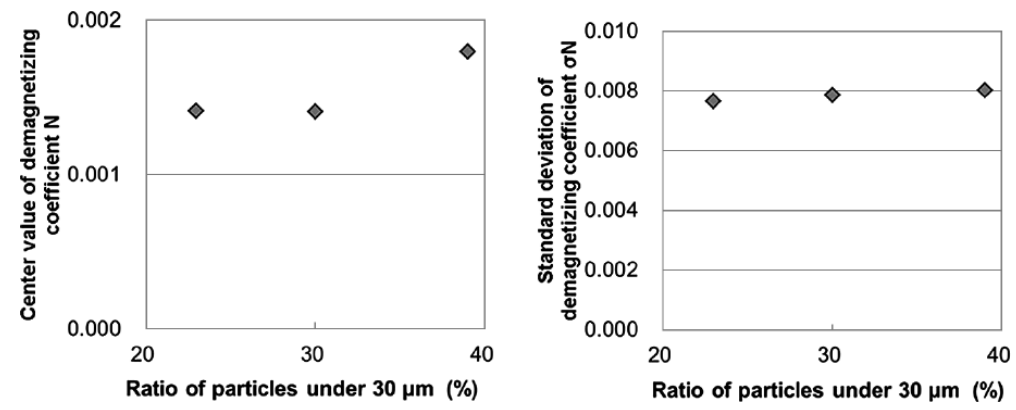

Fig. 4 The relationship between the ratio of particles under $30 \mu \mathrm{m}$, and the center values and the standard deviations. 


\section{References}

1) T. Ishimine, et al.: "Development of FeSiAl-Based Low Iron Loss Soft Magnetic Powder Cores", Proceedings of the 2014 World Congress on Powder Metallurgy \& Particulate Materials, (2014) 09-189-09-199.

2) S. Takashiro, et al.: "Analysis of High Frequency Magnetic Properties of Compressed Iron Powder Cores”, J. Jpn. Soc. Powder Powder Metallurgy, 32 (1985) 259-263.
3) T. Saito, et al.: "Initial Magnetization Curves for Powder Cores Taking into Account Distribution of Demagnetizing Field”, J. Magn. Soc. Jpn., 33 (2009) 434-440.

4) M. T. Johnson, et al.: "A coherent model for the complex permeability in polycrystalline ferrites", IEEE Trans. Magn., 26 (1990) 1897-1989.

5) K. Ota: Jikikogaku no kiso I, kyoritsu zensyo (1973) 35. 